\title{
Wie krisenfest sind betriebliche Bündnisse zur Beschäftigungssicherung?
}

\author{
Claudia Bogedan \\ Wolfram Brehmer \\ Hartmut Seifert
}

\begin{abstract}
Der Arbeitsmarkt in Deutschland hat den Kriseneinbruch im Vergleich zu anderen Ländern relativ unbeschadet überstanden. Vom deutschen „Beschäftigungswunder“ ist die Rede. Dieses Phänomen ist nicht zuletzt auch Ergebnis von betrieblichen Bündnissen für Arbeit. Sie gewähren den Arbeitnehmern in schweren Zeiten Beschäftigungssicherheit und helfen den Arbeitgebern, das Arbeitsvolumen ohne Entlassungen zu reduzieren, Kosten zu senken und Umstrukturierungen zu organisieren. Während der Laufzeit ist es jedoch jederzeit möglich, dass Arbeitgeber ihre Zusagen nicht mehr einhalten. In welchem Umfang verstoßen Betriebe gegen die Vereinbarungen und welche Faktoren beeinflussen die Wahrscheinlichkeit, dass es hierzu kommt? ${ }^{1}$
\end{abstract}

\section{Problemstellung}

Betriebliche Bündnisse für Arbeit (BBA) haben sich in den letzten Jahren etabliert. Sie sollen konjunkturelle oder branchen- und unternehmensspezifische Problemlagen meistern helfen, die Wettbewerbsfähigkeit stärken und bedrohte Beschäftigungsverhältnisse sichern. Im Prinzip basieren sie auf einem Tausch zwischen Arbeitgebern und Arbeitnehmern: Die Beschäftigten machen Zugeständnisse bei Arbeitszeit und Einkommen, während der Arbeitgeber im Gegenzug Beschäftigungs- oder Standortsicherungsgarantien zusagt. Die Beschäftigten gewinnen für eine vereinbarte Zeit Arbeitsplatz- und Einkommenssicherheit, der Arbeitgeber reduziert Arbeitskosten und Arbeitseinsatz. Diese Beidseitigkeit kennzeichnet unabhängig davon, ob es sich um einen Äquivalententausch handelt - in formaler Hinsicht betriebliche Bündnisse zur Beschäftigungssicherung bzw. für Arbeit. ${ }^{2}$

BBA stellen einen Kompromiss in einer für beide Betriebsparteien bedrohlichen Lage dar. Die beteiligten Parteien verpflichten sich vertraglich, die von ihnen gemachten Zugeständnisse in entsprechende Handlungen umzusetzen. Im Unterschied zu sonstigen Vereinbarungen liegt deren Durchführung allein in den Händen des Arbeitgebers, dem die Leitung des Betriebs obliegt. Hinzu kommt, dass die vereinbarten Zusagen nicht zeitgleich Zug um Zug, sondern asynchron erfüllt werden. Während die Zusagen der Beschäftigtenseite in der Regel unmittelbar realisiert werden, weisen die des Arbeitgebers in die Zukunft und stehen deshalb unter größeren Unsicherheiten. $\mathrm{Ob}$ ein Arbeitgeber die $\mathrm{Zu}-$ sage, für einen definierten Zeitraum auf betriebsbedingte Kündigungen zu verzichten oder die Beschäftigung auf dem gegebenen Niveau zu stabilisieren, auch tatsächlich einhält, bleibt bis zum Ablauf der vereinbarten Vertragsdauer ungewiss. Deshalb stellt sich die Frage, ob die vereinbarten Zusagen überhaupt eingehalten werden und unter welchen Bedingungen von Arbeitgebern gemachte Zusagen zur Beschäftigungssicherung nicht oder nur teilweise erfüllt werden. Dieser Frage nach der Stabilität von Zusagen zur Beschäftigungssicherung geht dieser Beitrag nach.

Die Fragestellung erscheint deshalb besonders relevant, weil die Rezession 2008/09 den vorangegangenen Konjunkturaufschwung ziemlich abrupt beendete und rückläufige Aufträge und Umsätze viele Betriebe mit bislang unbekannter Schärfe überrascht hatten. Das Bruttoinlandsprodukt ging in nur kurzer Zeit um insgesamt 6,2 \% zurück (Herzog-Stein/ Seifert 2010). Die Betriebe mussten kurzfristig reagieren. Nicht absehbar war, wie tief und lang die Krise sein würde. Angesichts dieser Unsicherheiten war nicht auszuschließen, dass bestehende Verein- barungen über Beschäftigungssicherung unter Druck geraten und infrage gestellt würden. Die Konjunkturprognosen ließen nicht erkennen, dass die Nachfrage nach Gütern und Dienstleistungen nach dem scharfen Konjunktureinbruch rasch wieder anziehen würde. Der Abschluss von neuen Beschäftigungsvereinbarungen im Verlauf der Krise erfolgte also - insbeson-

\footnotetext{
1 Wir danken Alexander Herzog-Stein sowie dem Koordinator des Schwerpunktheftes für die kritische Begutachtung und hilfreiche Überarbeitungshinweise. Zudem danken wir Martin Behrens, Reinhard Bispinck und Manuela Maschke für ihre fachkundige Beratung über betriebliche Bündnisse für Arbeit und Kollektivverhandlungen. 2 Beide in der Literatur üblichen Begriffe werden nachfolgend synonym verwendet.
}

Claudia Bogedan ist Wissenschaftlerin in der Abteilung Wirtschafts- und Sozialwissenschaftliches Institut in der Hans-Böckler-Stiftung (WSI). Arbeitsschwerpunkte: International vergleichende Arbeitsmarktpolitik, soziale Sicherung flexibler Erwerbsbiografien. e-mail: claudia.bogedan@boeckler.de Wolfram Brehmer ist Wissenschaftler im WSI. Arbeitsschwerpunkte: Betriebs- und Personalräteforschung. e-mail:wolfram-Brehmer@boeckler.de Hartmut Seifert, Dr., ehemaliger Leiter der Abteilung WSI in der Hans-Böckler-Stiftung. Arbeitsschwerpunkt: Arbeitszeit- und Arbeitsmarktforschung. e-mail: H.G.Seifert@t-online.de 
dere auch für die Beschäftigten - unter erhöhter Unsicherheit. Unsicher war aber auch, ob die bereits vor Beginn der Krise vereinbarten BBA angesichts der veränderten Wirtschaftslage unverändert fortbestehen würden.

Wenig ist bislang über die Einhaltung von Bündniszusagen bekannt (MassaWirth 2007) und noch weniger über betriebliche Bündnisse in der Weltwirtschaftskrise. Die vorliegenden Analysen beziehen sich überwiegend auf BBA während der Rezession 2001 - 2005 (SVR 2007). Insofern betreten wir wissenschaftliches Neuland. Dieser Beitrag untersucht auf Basis der WSI-Betriebsrätebefragungen der Jahre 2009 und 2010, welche Faktoren das Risiko beeinflussen, dass Betriebe ihre Zusagen zur Beschäftigungssicherung nicht einhalten. Unter welchen Bedingungen brechen die Arbeitgeber ihre Zusage?

Ausgehend von einem kurzen Überblick zum Forschungsstand bilden wir unsere Hypothesen darüber, welche Faktoren die Brechungswahrscheinlichkeit von Beschäftigungssicherungsvereinbarungen bedingen (Abschnitt 2). In Abschnitt 3 folgen Informationen über die Datenquelle sowie zum methodischen Vorgehen, bevor erste Befunde über BBA während der jüngsten Wirtschaftskrise dargestellt werden (Abschnitt 4). Zwei Regressionsanalysen zum Hypothesentest bilden den Kern von Abschnitt 5. Ein kurzes Fazit rundet den Beitrag ab (Abschnitt 6).

\section{Forschungsstand und Hypothesen}

Betriebliche Bündnisse, die darauf zielen, als Gegenleistung für Konzessionen der Beschäftigten bei Lohn oder Arbeitszeit Beschäftigung zu sichern, Standortverlagerungen, Auslagerungen oder Kündigungen zu verhindern und die Wettbewerbsfähigkeit zu steigern, haben sich mittlerweile im System der industriellen Beziehungen etabliert. Sie basieren häufig auf tarifvertraglichen Öffnungs- und Differenzierungsklauseln und bieten den betrieblichen Akteuren neue Möglichkeiten, die kostenund beschäftigungsrelevanten Parameter Einkommen und Arbeitszeit dezentral im Rahmen tariflicher Rahmenvorgaben zu variieren. Ihre Rückwirkungen auf das Tarifvertragssystem und die Beziehung zwi- schen Arbeitgebern und Arbeitnehmern sind Gegenstand zahlreicher Untersuchungen (Büttner/Kirsch 2005; Hassel/Rehder 2001; Kaden 2003).

\subsection{BETRIEBLICHE ARBEITS- BEZIEHUNGEN UND BBA}

Der Abschluss von betrieblichen Bündnissen setzt voraus, dass Arbeitgeber und Arbeitnehmer einen Teil ihrer antagonistischen Interessen aufgeben und sich "zusammenraufen“. Hassel und Rehder (2001) zeigen, dass gerade für größere Betriebe friedliche Arbeitsbeziehungen einen Effizienz steigernden Effekt in der Arbeitsorganisation entfalten. Allerdings ist diese Form des Co-Managements von Betriebsräten nicht folgenlos für die Beziehung zwischen Beschäftigten und ihrer Interessenvertretung (Rehder 2006). Demnach entstehen insbesondere in Betrieben mit langjähriger Bündnispraxis Abnutzungserscheinungen und Repräsentationsdefizite gegenüber den Beschäftigten.

In einer jüngeren Studie untersuchten Nienhüser und Hoßfeld (2010a, 2010b), wie sich das Verhältnis zwischen Management und Betriebsrat auf BBA auswirkt. Sie kommen zu dem Schluss: Je vertrauter die Beziehung zwischen Betriebsrat und Management, desto eher sind betriebliche Interessenvertretungen geneigt, Absprachen mit dem Management zu treffen. Zur Erfassung des Vertrauens zwischen den Betriebsparteien messen die Autoren unter anderem die Zustimmung zu der Aussage: „Das Management/der Betriebsrat hält sich an Vereinbarungen“. Verlässlichkeit kann demnach als wichtige Bedingung für das Verhältnis der Betriebsparteien gesehen werden. Wie bei den meisten sozialen Interaktionen ist davon auszugehen, dass Verlässlichkeit und Vertrauen sich wechselseitig verstärken. Für unsere Fragestellung erwarten wir folglich einen signifikanten Einfluss vertrauensvoller Beziehungen der Betriebsparteien auf die Wahrscheinlichkeit, dass der Arbeitgeber seine Zusagen einhält. Hypothese 1 lautet: Je konfliktärmer die Beziehung zwischen Management und Betriebsrat, desto stabiler sind Absprachen zwischen ihnen. Wird die Mitwirkung des Betriebsrats durch den Arbeitgeber nie behindert, sinkt die Wahrscheinlichkeit, dass der Arbeitgeber seine Zusagen nicht einhält.

\subsection{INHALTE BETRIEBLICHER BÜNDNISSE UND IHRE BEDEUTUNG}

Ein anderer Zweig der Literatur befasst sich mit der Verbreitung betrieblicher Bündnisse und ihrer Inhalte (Seifert 2002; Seifert/ Massa-Wirth 2004) sowie ihren Wirkungen auf das Tarifvertragssystem. Eine umfassende Studie von Rehder (2003) zeigt dabei, dass die neuen Bündnisse das Flächentarifvertragssystem zwar konvertiert, aber nicht ausgehöhlt haben. Massa-Wirth (2007) sieht im Gegensatz zu Rehder je nach den vereinbarten inhaltlichen Schwerpunkten systemgefährdende Risiken für das Tarifvertragssystem. Während Vereinbarungen über flexible Arbeitszeiten, die Modernisierung der Arbeitsorganisation oder die Qualifizierung der Beschäftigten, die primär der Produktivitätssteigerung dienen, die lohnpolitische Kartellfunktion des Flächentarifes nicht infrage stellen, befürchtet er, dass Konzessionen beim Einkommen einen Paradigmenwechsel von einer wettbewerbsbegrenzenden hin zu einer wettbewerbsorientierten Tarifpolitik fördern könnten. Insofern kommt den vereinbarten Regelungsinhalten eine zentrale Bedeutung für die Systemwirkungen zu. Außerdem findet der Autor einen Zusammenhang zwischen unterschiedlichen Inhalten von Arbeitgeberzusagen und der Häufigkeit, dass diese nicht eingehalten werden. Das führt er zurück auf unterschiedliche Sanktionspotenziale bei Nicht-Einhaltung bei den jeweiligen Zusagen. So verfügen Arbeitnehmer nur im Fall des Ausschlusses betriebsbedingter Kündigungen über die Möglichkeit, die Einhaltung vor dem Arbeitsgericht einzuklagen. Insgesamt wurde in der von Massa-Wirth untersuchten Stichprobe in $9 \%$ der Bündnisbetriebe von einer oder mehreren Zusagen arbeitgeberseitig abgewichen (Massa-Wirth 2007, S. 117). Diese Angaben sind allerdings mit den unsrigen nur schwer vergleichbar, da die BBA jeweils anders operationalisiert wurden.

Wir analysieren auch, ob die unterschiedlichen Arbeitnehmerzugeständnisse - von Einschnitten beim Entgelt bis hin zur Kurzarbeit - einen Effekt auf die Einhaltung von Zusagen haben. Ein eindeutiger Wirkungszusammenhang ist allerdings aus der Literatur nur schwer ableitbar. Einerseits ist davon auszugehen, dass schmerzhafte Zugeständnisse der Beschäftigten wie z.B. Lohneinbußen nur dann Teil des Bündnisses werden, wenn 
andere Möglichkeiten bereits ausgeschöpft wurden und die schwierige wirtschaftliche Lage des Betriebes einen solchen Schritt für die Beschäftigten erforderlich macht. Andererseits könnten besonders schmerzhafte Einschnitte das Verhandlungsergebnis einer ausgeprägt asymmetrischen Beziehung zwischen Arbeitgeber und Betriebsrat sein, in der der Betriebsrat übervorteilt wurde. Stehen Zugeständnisse der Beschäftigten und Zusagen der Arbeitgeber nämlich in einem ungleichen Verhältnis zueinander, so dürfte sich dies nachteilig auf die betrieblichen Beziehungen auswirken, nicht nur zwischen Betriebsrat und Betriebsleitung, sondern auch zwischen Betriebsrat und Beschäftigten. Deshalb (vgl. Hypothese 1) dürfte die Instabilität der Vereinbarungen zunehmen, je ungleicher die Inhalte ausbalanciert sind (Hypothese 2).

Sämtliche Arbeitgeberzusagen, vom Ausschluss betriebsbedingter Kündigungen bis zur Übernahme von Auszubildenden, zielen auf mittelbare oder direkte Beschäftigungseffekte. Wir untersuchen den Einfluss der unterschiedlichen Arbeitgeberzusagen auf die Stabilität derselben. Dabei vermuten wir in Anlehnung an Massa-Wirth, dass je größer die Kontroll- und Sanktionsmöglichkeiten der Arbeitnehmerseite sind, wie beispielsweise im Falle der Nicht-Einhaltung des Ausschlusses betriebsbedingter Kündigungen, desto geringer wird die Wahrscheinlichkeit, dass Versprechen gebrochen werden (Hypothese 3).

Allerdings zeigen Studien, die den beschäftigungspolitischen Erfolg der Bündnisse messen, dass Bündnisbetriebe eher als solche ohne einen Beschäftigungsabbau vollzogen haben (Hübler 2005; Bellmann et al. 2008). Denn auch die Zusage eines Ausschlusses betriebsbedingter Kündigungen bedeutet nicht zwangsläufig, dass es zu keinerlei Veränderungen im Personalbestand kommt. Leider liefern die Befunde der beiden Studien keinerlei Hinweise auf unsere Forschungsfrage nach dem Bruch von Arbeitgeberzusagen. Die Arbeitgeber können alle gemachten Versprechen erfüllen und dennoch in der Gesamtbilanz Beschäftigung abbauen, z.B. durch Ausnutzen von Fluktuation.

\subsection{AUSGANGSLAGE UND KONTEXT- BEDINGUNGEN}

In der Literatur werden unterschiedliche Anlässe oder Motive für Bündnisse unterschieden. Primär richten sich die Bünd- nisse entweder auf eine Verbesserung der Stellung gegenüber Wettbewerbern oder sind Reaktion auf wirtschaftlich schwierige Zeiten. Es lassen sich folglich Krisenbündnisse, „in denen die Arbeitsplätze durch einen negativen Technologie- oder Nachfrageschock dauerhaft unter Anpassungsdruck geraten“ (Berthold et al. 2003, S. 177) von Innovations- oder Wettbewerbsbündnissen unterscheiden, bei denen es den Unternehmen primär darum geht, sich an Marktveränderungen anzupassen, sei es aufgrund einer gestiegenen Internationalisierung, neuer Kundenbedürfnisse oder eines Technologiewandels. Die Situation, in der sich der Betrieb befindet, hat - so vermuten wir - auch Einfluss auf die Stabilität der Bündnisse. Wir unterscheiden in Anlehnung an Berthold et al. (2003) Betriebe, die zum Befragungszeitpunkt von der Wirtschaftskrise oder einer Unternehmenskrise betroffen waren, von denen, deren Bündnis entweder aus einer früheren Krise fortbesteht oder als Innovations- oder Wettbewerbsbündnis bezeichnet werden könnte.

Insbesondere die schwere Rezession dürfte, wie in der Einleitung dargelegt, als externer Schock auf Betriebe und bereits bestehende Bündnisse gewirkt haben. $\mathrm{Hy}$ pothese 4 lautet, dass in krisenbetroffenen Betrieben Zusagen eher nicht eingehalten werden. Haben die Betriebsparteien allerdings die Vereinbarungen anlässlich der Krise verändert oder überhaupt erst abgeschlossen, so sinkt das Brechungsrisiko, da diese Bündnisse bereits die veränderten Rahmenbedingungen reflektieren (Hypothese 5). Beinhaltet die Vereinbarung darüber hinaus von vornherein eine Notfallklausel, d.h. einen Vorbehalt, der ein Abweichen von der Vereinbarung in Notsituationen erlaubt, steigt die Wahrscheinlichkeit, dass die Zusagen des Arbeitgebers nicht eingehalten werden, da die Notfallklausel geradezu dazu einlädt (Hypothese 6).

Aus früheren Untersuchungen ist $\mathrm{zu}$ dem bekannt, dass die Existenz eines BBA Flexibilität unterhalb des Flächentarifvertrages gewährt und den Arbeitgebern Instrumente interner Flexibilität eröffnet (Bellmann et al. 2008). Handelt es sich bei den BBA um Tarifabweichungen, die die Tarifnormen unterschreiten, werden diese entweder als betriebsbezogene Ergänzungstarifverträge oder Betriebsvereinbarungen überwiegend mit Zustimmungspflicht der Tarifparteien geregelt (Haipeter 2009).
Somit sind die Gewerkschaften direkt involviert. Im Unterschied zum Betriebsrat nehmen Gewerkschaften eher eine überbetriebliche Perspektive ein und verfügen über mehr Verhandlungsmacht. ${ }^{3}$ Somit gehen wir in Hypothese 7 davon aus, dass das gewerkschaftliche Zustimmungserfordernis die Durchsetzung der Interessen der Arbeitgeber erschwert und diese folglich eher dazu tendieren, die Vereinbarungen zu verletzen.

Neben der Ausgangssituation des Bündnisses und dessen Rückbindung an das Tarifvertragssystem bildet die Form, in der das Bündnis beschlossen wird, eine wichtige Kontextbedingung, die Einfluss auf die Stabilität und Verlässlichkeit der Arbeitgeberzusagen haben dürfte. Grundsätzlich ist es den Betriebsparteien nämlich überlassen, in welcher Form sie Bündnisse abschließen. Neben schriftlichen Betriebsvereinbarungen kommen auch schriftliche oder mündliche Regelungsabreden infrage. Mündliche Absprachen bieten sich insbesondere dann an, wenn die Betriebsparteien auf eine kurzfristig eingetretene Situation schnell reagieren müssen. Allerdings unterscheidet sich die Schriftform insofern von der mündlichen, als Tarifverträge und Betriebsvereinbarungen unmittelbar Wirkung entfalten. Konkret bedeutet dies, dass sowohl die Regelungen des Tarifvertrags als auch der Betriebsvereinbarungen direkt die geltenden Regeln der individuellen Beschäftigungsverhältnisse verändern. Für die Arbeitgeber ergibt sich hieraus ein Rationalisierungsgewinn, da nicht mit allen Beschäftigten separat eine individuelle Vereinbarung geschlossen bzw. der Arbeitsvertrag geändert werden muss. Deshalb gehen wir davon aus, dass die Form der Vereinbarungen nicht nur von den Inhalten abhängt, sondern auch einen Einfluss auf die Stabilität der Vereinbarungen ausübt. Die Schriftform dürfte eine höhere Verbindlichkeit erzeugen (Hypothese 8), denn einerseits handelt es sich bei erfolgreichen Pakten um komplexe Regelungspakete (Seifert 2002), bei denen sich allein aus Praktikabilitätserwägungen die Schriftform empfiehlt. Andererseits kann eine schriftliche Vereinbarung auch leichter von der Arbeitnehmervertretung eingefordert werden. Zudem sind

3 Diese ergibt sich aus der Tatsache, dass Gewerkschaften im Gegensatz zum Betriebsrat nicht dem Interesse des Betriebes verpflichtet sind und über andere Mobilisierungsmöglichkeiten verfügen. 
Betriebsvereinbarungen und Tarifverträge transparenter, da sie für alle Beschäftigten einsehbar sein müssen. Die Transparenz vorhandener Rechte unterstützt die Mobilisierung der Rechtsansprüche durch die Arbeitnehmer (Kocher 2009); nur wer sein Recht kennt, kann es auch einfordern. Allerdings gehen wir bei Tarifverträgen davon aus, dass diese seltener eingehalten werden (Hypothese 9). Denn tarifliche Regelungen bedürfen, auch wenn es sich um Firmentarife auf Unternehmensebene handelt, immer einer betrieblichen Umsetzung. Dabei unterliegen sie per se einer Interpretation der Betriebsparteien, zumal die zu einem Unternehmen gehörenden Betriebe unterschiedliche Problemkonstellationen aufweisen können. Außerdem ist die Distanz zu Regelungen höher, wenn sie von Dritten abgeschlossen wurden (Rottleuthner 1987). Auch können Gewerkschaften aus der Distanz schwerer kontrollieren, ob die Vereinbarungen auch eingehalten wurden.

Um weitere relevante Faktoren für eingehaltene betriebliche Bündnisse $\mathrm{zu}$ erfassen, ergänzen wir einige Kontrollvariablen, um strukturelle und sonstige erklärende Faktoren zu berücksichtigen. Dazu gehören die Tarifbindung, die Lage (Ost- vs. Westdeutschland), die Exportabhängigkeit sowie BetriebsgröBe und Branche. Beispielsweise zeigen Nienhüser und Hoßfeld (2010b), dass für Betriebsräte im industriellen Sektor eine statistisch signifikant höhere Neigung zur Verlagerung von Verhandlungen auf die Betriebsebene besteht. Dies sind zugleich jene Sektoren, die von der Krise am stärksten betroffen waren (Bogedan et al. 2009). Mit wachsender Größe des Betriebs verändern sich die Arbeitsbeziehungen. In aller Regel steigt angesichts größerer Ressourcenausstattung die Professionalität im Personalmanagement und in der betrieblichen Interessenvertretung. Darüber hinaus sind Skaleneffekte und damit Rationalisierungsgewinne für die Arbeitgeber über betriebliche Bündnisse in großen Betrieben höher (vgl. zum Zusammenhang von Betriebsgröße und Effizienzgewinnen Hassel/Rehder (2001)). Auch tendieren große Betriebe zu betrieblichen Lösungen, die es ihnen erlauben, flexibel auf exogene Schocks zu reagieren (Bellmann et al. 2008).

Wie wir bei der Überprüfung dieser Hypothesen vorgegangen sind, erläutert der folgende Abschnitt.

\section{Methode und Vorgehen}

Zwischen Juli und September 2009 führte das WSI eine telefonische Befragung von Betriebsräten durch, um betriebliche Aktivitäten zur Beschäftigungssicherung während der Wirtschaftskrise zu erforschen (Bogedan et al. 2009). Diese Befragung war die dritte Welle eines 2007 gestarteten Panels. $\mathrm{Zu}$ Panelbeginn wurden die Betriebe durch eine disproportional geschichtete Zufallsziehung aus der Betriebsdatei der Bundesagentur für Arbeit gewählt. Alle in diesem Beitrag gemachten Angaben sind daher gewichtet, ${ }^{4}$ um die Disproportionalität der Stichprobe sowie die Panelmortalität durch die vorangegangen Wellen auszugleichen. Die Stichprobe erlaubt repräsentative Aussagen für privatwirtschaftliche Betriebe ab 20 Beschäftigten und mit einem Betriebsrat. ${ }^{5}$

Mit einer Ausnahme stammen alle in diesem Artikel verwendeten Angaben aus der Betriebsrätebefragung des Jahres 2009. Lediglich die Information, ob innerhalb eines BBA alle Arbeitgeberzusagen eingehalten wurden oder nicht, stammt nicht nur aus der 2009er Erhebung, sondern auch aus der Folgebefragung im Frühjahr 2010. Dieses Vorgehen erscheint uns sinnvoll, da besonders bei den anlässlich der Wirtschaftskrise veränderten oder neu abgeschlossenen Bündnissen ein gewisser zeitlicher Abstand sinnvoll ist, bevor geprüft wird, ob ein Bündnis erfüllt wurde oder nicht. Daher treffen wir nur Aussagen auf Basis der Betriebe, die sowohl im Jahr 2009 als auch im Jahr 2010 an der WSI-Betriebsrätebefragung teilnahmen $(\mathrm{n}=1.738)$.

In einem ersten Schritt beschreiben wir für diese Untersuchungspopulation, welche Betriebe in welchem Umfang BBA während der Wirtschaftskrise nutzten (Abschnitt 4.1). In einem zweiten Schritt grenzen wir unsere Untersuchungspopulation auf die Bündnisbetriebe ein $(\mathrm{n}=1.076)$, um sie untereinander zu vergleichen und deren Inhalte zu ermitteln (Abschnitt 4.2 und 4.3).

Nach dem deskriptiven Teil stellen wir zwei logistische Regressionsmodelle vor (Abschnitt 5). Diese gehen der Frage nach, welche Faktoren in welchem Maße die Wahrscheinlichkeit des arbeitgeberseitigen Bruchs von betrieblichen Beschäftigungsbündnissen bedingen. Das erste Regressionsmodell umfasst alle Bündnisbetriebe, ${ }^{6}$ das zweite Regressionsmodell lediglich Betriebe mit schriftlichen Vereinbarungen, welche von der Wirtschaftskrise oder einer sonstigen Betriebskrise betroffen sind ( $\mathrm{n}=291$ ). Diesen Zuschnitt wählen wir, da wir aufgrund der Schriftform von anderen Wirkungszusammenhängen als bei mündlichen Vereinbarungen ausgehen (vgl. Abschnitt 2). Außerdem analysieren wir die Eigenschaften der schriftlichen Vereinbarungen selbst. In Anlehnung an Berthold et al. (2003) fassen wir zudem alle Krisenbetriebe in einer Kategorie zusammen und schließen damit Wettbewerbsbündnisse aus der Analyse aus. Letztere werden aufgrund anderer Motive geschlossen, was sich in unterschiedlichen inhaltlichen Vereinbarungen zwischen Belegschaft und Geschäftsführung niederschlägt.

Die in Abschnitt 2 formulierten Hypothesen für die Regressionsmodelle haben wir wie folgt operationalisiert: Die Betriebsräte wurden in den Befragungen der Jahre 2009 und 2010 gefragt, ob ihr „Betrieb alle beschäftigungsrelevanten $\mathrm{Zu}$ sagen eingehalten hat". Die Information bildet die dichotome abhängige Variable. Wurde von einer Nichteinhaltung entweder 2009 oder 2010 berichtet, gehen wir von einem bis zum Befragungszeitpunkt verletzten Bündnis aus. Das ist in rund 29 \% der Bündnisbetriebe der Fall.

Sämtliche erklärenden Variablen stammen aus der 2009er Befragung: Die Beschäftigtenzahl wird inklusive Leiharbeitnehmer numerisch in das Modell eingeführt. Alle anderen unabhängigen Variablen sind dagegen dummykodiert. Die Angabe zur Tarifgebundenheit fasst Firmen- und Flächentarife zusammen und stellt sie den nicht tarifgebunden Betrieben gegenüber. Die Lage des Betriebs ist differenziert nach Ost- und Westdeutschland, die Branchen sind in sechs Gruppen zusammengefasst. Die Exportabhängigkeit wurde mit vier Ausprägungen erhoben, von denen jeweils zwei zusammengefasst wurden: „keine Exporte“ und „leichte Exportabhängigkeit“ gegenüber „mittel“ und „stark exportabhängig“. Die betriebliche Krisenbetroffenheit wurde mit

4 Horvitz-Thompson-Quotientenschätzer. Nicht gewichtet sind Fallzahlen und Regressionsschätzungen

5 Für weitere Informationen siehe Schäfer (2008).

6 Die Fallzahl sinkt gegenüber allen Bündnisbetrieben (1.076) im deskriptiven Teil auf 1.016 Betriebe, da Betriebe mit fehlenden Angaben bei einer beliebigen Frage aus der Regression ausgeschlossen wurden. 
zwei direkten Einschätzungsfragen erhoben: „Ist Ihr Betrieb seit Anfang Juli 2008 von der Weltwirtschaftskrise betroffen?“ und „Leidet Ihr Betrieb seit Anfang Juli 2008 unter Krisenlagen, die unabhängig von der allgemeinen Wirtschaftskrise entstanden sind?" Aus der Kombination dieser beiden Fragen ergeben sich vier Fälle, die dummykodiert in die Regressionen aufgenommen werden. Die Schriftform der Vereinbarung wurde differenziert nach Betriebsvereinbarung, tariflicher Regelung und sonstiger Regelungsabrede erhoben. Mündliche Vereinbarungen liegen dann vor, wenn keine schriftlichen Vereinbarungen angegeben wurden, aber sowohl Arbeitgeberzusagen zur Beschäftigungssicherung als im Gegenzug auch Arbeitnehmerzugeständnisse beobachtet werden. Die Beziehung zwischen Arbeitgeber und Betriebsrat wird durch die Einschätzungsfrage abgebildet, wie häufig es zu Behinderungen der betriebsrätlichen Mitwirkung durch den Arbeitgeber kommt. Die inhaltlichen Beschäftigungssicherungszusagen des Arbeitgebers wurden durch zehn Einzelfragen erhoben und werden inhaltlich zusammengefasst über sechs dummycodierte Variablen aufgenommen. Gleiches gilt für die Arbeitnehmerzugeständnisse. Bei diesen sowie den Arbeitgeberzusagen handelt es sich um Mehrfachnennungen. Ob ein $\mathrm{Zu}$ stimmungserfordernis der Gewerkschaften nötig war, wurde direkt erfragt, ebenso das Vorhandensein und die Nutzung einer Notfallklausel. Für die Symmetrie des Tauschs zwischen Arbeitgeber und Arbeitnehmer verwenden wir zwei dummycodierte Einstellungsfragen an den Betriebsrat. Zu bewerten waren die Aussagen: „Arbeitgeber und Belegschaft mussten jeweils einen „fairen" Preis für die Vereinbarung zahlen" und „Diese Vereinbarung ist von der Belegschaft zu teuer erkauft worden“.

\section{4 \\ Verbreitung und Inhalte der Bündnisse}

\subsection{VERBREITUNG}

Management und Betriebsrat haben in $58,5 \%$ aller befragten Betriebe ein Bündnis zur Beschäftigungssicherung vereinbart. Der hohe Anteil erklärt sich durch die Situation der Weltwirtschaftskrise. Das zeigt sich deutlich bei der Betrachtung nach Krisenbetroffenheit: Von der Wirtschaftskrise betroffene Betriebe sind zu 70 \% Bündnisbetriebe, ohne lediglich zu $46 \%$. Zudem geht der hohe Anteil auf die hier verwendete Definition zurück. Denn wir erfassen nicht nur Betriebsvereinbarungen oder schriftliche Regelungsabreden, sondern auch informelle Absprachen mit mündlichen Arbeitgeberzusagen. Der Verzicht auf die Schriftform dürfte mitunter damit zu tun haben, dass die für viele Betriebe plötzlich hereingebrochene Wirtschaftskrise kurzfristige Reaktionen erzwang.

Der Anteil der Bündnisbetriebe steigt mit der Betriebsgröße; er streut zudem stark zwischen den Wirtschaftsbereichen. Die Anteilswerte bewegen sich zwischen $30 \%$ im Bereich Kredit und Versicherungen und $78 \%$ im Investitions- und Gebrauchsgütergewerbe. Hierin spiegelt sich mitunter die ungleiche Betroffenheit der Branchen von der Wirtschaftskrise.

Betrachtet man ausschließlich Bündnisbetriebe, dann sehen sich knapp die Hälfte von der Weltwirtschaftskrise betroffen, $9 \%$ von einer sonstigen betrieblichen Krisenlage und $14 \%$ nennen beide Antwortkategorien und $28 \%$ verneinen eine Krisenbetroffenheit. Diese BBA stammen entweder aus frü- heren krisenhaften Situationen oder sind als Innovations- oder Wettbewerbsbündnisse einzustufen (Berthold et al. 2003).

Mündliche Vereinbarungen überwiegen mit einem Anteil von $69 \%$, die restlichen Betriebe verteilen sich auf verschiedene Formen schriftlicher Vereinbarungen. Größere Betriebe tendieren eher zu schriftlichen Vereinbarungen, ebenso tarifgebundene Betriebe. Diese zwei Merkmale sind zwar untereinander korreliert, da große Betriebe häufiger einer Tarifbindung unterliegen. Doch auch bei gegenseitiger Kontrolle bleibt ein autarker Befund bezüglich der Verteilung mündlich/schriftlich bestehen.

Die schriftlichen Vereinbarungen lassen sich differenzieren zwischen Betriebsvereinbarungen (23\%), tariflichen Regelungen (13\%) und sonstigen schriftlichen Regelungsabreden (4\%), wobei ein Betrieb parallel auch über mehrere schriftliche Vereinbarungen verfügen kann. Unter allen Betrieben mit schriftlichen Vereinbarungen änderten rund $45 \%$ ihre Vereinbarungen in Reaktion auf die Krise oder schlossen neue ab.

\subsection{INHALTE}

Betrachtet man in den beidseitigen Vereinbarungen zunächst die von den Beschäftigten gemachten Zugeständnisse, dann dominieren (in 77,5 \% der Bündnisbetriebe) Regelungen, die sich auf die Gestaltung der Arbeitszeit beziehen (Zapf/Brehmer 2010). Personelle oder organisatorische Maßnahmen (z.B. Ver- und Umsetzungen) folgen mit deutlichem Abstand (47 \%). Monetäre Konzessionen (Abstriche beim Entgelt und Einschnitte bei den betrieblichen Sozialleistungen) sind Regelungsgegenstand in einem knappen Viertel (24 \%) aller Bündnisse (Tabelle 1).

\section{Tabelle 1: Umgesetzte oder geplante Maßnahmen zur Beschäftigungssicherung bei Konzessionen}

der Beschäftigten - in \% -

\begin{tabular}{|c|c|c|c|c|}
\hline & \multicolumn{4}{|c|}{ Betriebe mit Bündnissen für Arbeit } \\
\hline & $(n=1076)$ & $\begin{array}{l}\text { davon nur mündliche } \\
\text { Arbeitgeberzusage } \\
(n=662)\end{array}$ & $\begin{array}{l}\text { davon schriftliche } \\
\text { Arbeitgeberzusage und } \\
\text { krisenbetroffen } \\
(n=414)\end{array}$ & $\begin{array}{c}\text { von diesen } 414 \\
\text { verändert oder neu } \\
\text { geschlossen in Krise } \\
(n=149)\end{array}$ \\
\hline Abstriche beim Entgelt & 23,9 & 20,3 & 31,8 & 41,9 \\
\hline Kurzarbeit & 37,4 & 34,9 & 42,9 & 55,5 \\
\hline Abbau Arbeitszeitkonten / Aufbau Zeitschulden & 53,7 & 53,1 & 54,9 & 53,4 \\
\hline Veränderung sonstige Arbeitszeit & 16,3 & 14,8 & 19,7 & 22,2 \\
\hline Regelungen zur Urlaubsnahme & 24,3 & 21,1 & 31,2 & 42,8 \\
\hline Personelle und organisatorische Maßnahmen & 47,0 & 44,4 & 52,6 & 58,6 \\
\hline Einschnitte bei betrieblichen Sozialleistungen & 11,8 & 11,5 & 12,3 & 14,9 \\
\hline
\end{tabular}




\begin{tabular}{|c|c|c|c|c|}
\hline & \multicolumn{4}{|c|}{ Betriebe mit Bündnissen für Arbeit } \\
\hline & $(n=1076)$ & $\begin{array}{l}\text { davon nur mündliche } \\
\text { Arbeitgeberzusage } \\
\qquad(n=662)\end{array}$ & $\begin{array}{l}\text { davon schriftliche } \\
\text { Arbeitgeberzusage und } \\
\text { krisenbetroffen } \\
(n=414)\end{array}$ & $\begin{array}{c}\text { von diesen } 414 \\
\text { verändert oder neu } \\
\text { geschlossen in Krise } \\
\quad(n=149)\end{array}$ \\
\hline Ausschluss betriebsbedingter Kündigungen & 49,4 & 38,9 & 72,4 & 76,4 \\
\hline Erhalt der gegenwärtigen Belegschaftsstärke & 67,1 & 64,6 & 72,6 & 73,6 \\
\hline Erhalt von Ausbildungskapazitäten & 54,4 & 51,2 & 61,3 & 58,6 \\
\hline Erhalt des Standorts & 77,0 & 75,3 & 80,7 & 80,0 \\
\hline Garantie von Produktlinien & 29,6 & 28,0 & 33,2 & 31,0 \\
\hline Verzicht auf Outsourcing & 26,0 & 22,2 & 34,2 & 43,6 \\
\hline Neueinstellungen & 28,7 & 27,3 & 31,9 & 23,6 \\
\hline Übernahme von Auszubildenden & 43,4 & 39,5 & 52,0 & 49,1 \\
\hline Investitionen am Standort & 53,5 & 53,4 & 53,7 & 46,1 \\
\hline sonstige Zusagen & 9,9 & 7,5 & 15,0 & 15,1 \\
\hline
\end{tabular}

Vergleicht man die nur mündlichen mit den schriftlichen Vereinbarungen, so fällt auf, dass in der zweiten Gruppe durchschnittlich relativ mehr Maßnahmen verabredet wurden. Dieser Befund ist jedoch über die Krisenbetroffenheit zu relativieren. Noch höher liegt die durchschnittliche Zahl vereinbarter Maßnahmen unter den Betrieben, welche ihr BBA anlässlich der Wirtschaftskrise neu abschlossen oder anpassten. Die Schriftform überwiegt auch bei Vereinbarungen über Abstriche beim Entgelt oder zur Urlaubsnahme im Unterschied zu Maßnahmen wie der Nutzung von Arbeitszeitkonten. Das kann angesichts der unmittelbaren Drittwirkung von Betriebsvereinbarungen nicht verwundern, da sie sonst mit jedem betroffenen Arbeitnehmer individuell vertraglich fixiert werden müsste. Hinzu kommt, dass zwar die Einführung einiger Maßnahmen (wie Arbeitszeitkonten) in aller Regel der Schriftform bedarf, ihre Nutzung in der Krisensituation jedoch keine erneute schriftliche Vereinbarung voraussetzt.

Die meisten Betriebe (rund $60 \%$ ) vereinbaren komplexe Maßnahmenpakete und nicht nur einzelne Anpassungsinstrumente. Im Durchschnitt enthalten die Vereinbarungen etwas über zwei Einzelmaßnahmen. Mit der Größe der Betriebe steigt der Umfang der Maßnahmenbündel.

Ein ähnlich komplexes Bild zeigt sich bei den Arbeitgeberzusagen zur Beschäftigungssicherung. Die meisten Vereinbarungen enthalten mehrere (im Mittel vier bis fünf) beschäftigungsrelevante Zusagen. Am häufigsten, in $69 \%$ der Fälle, wurde der Erhalt des Standorts versprochen, gefolgt von der beschäftigungspolitisch bedeutsameren Zusage, die gegenwärtige Belegschaftsstärke zu erhalten (60\%). Gut $44 \%$ der Betriebsräte geben an, dass ihr Arbeitgeber betriebsbedingte Kündigungen im Gegenzug zu den arbeitnehmerseitigen Zugeständnissen ausgeschlossen hat. Auf Rang sechs folgt mit 39 \% die Übernahme von Auszubildenden (Tabelle 2). ${ }^{7}$

Wie bei den Konzessionen der Beschäftigten scheint auch bei den Arbeitgeberzusagen die Form der Vereinbarungen einen Einfluss auf die Inhalte zu haben. Die Anzahl der verschiedenen Zusagen nimmt im Schnitt bei der Schriftform gegenüber der mündlichen Form pro Betrieb zu. Demgegenüber bestehen kaum Unterschiede in der durchschnittlichen Anzahl an $\mathrm{Zu}-$ sagen unter den Betrieben, welche ihren Pakt anlässlich der Wirtschaftskrise neu abgeschlossen oder verändert haben.

\subsection{NICHT EINGEHALTENE ARBEITGEBERZUSAGEN}

In knapp einem Viertel (24 \%) aller Bündnisbetriebe haben die Arbeitgeber Elemente ihrer Zusagen nicht eingehalten. In $5 \%$ der Fälle kam es zu einem Bruch aller Zusagen. Diese Befunde lassen sich in Ermangelung von Vergleichswerten schwer bewerten. Zumindest muss der Gesamtanteil von $29 \%$, der hier als „Bruch einer Arbeitgeberzusage" bezeichnet wird, durch den Umstand relativiert werden, dass einerseits bereits eine beliebige nicht erfüllte Zusage als Nichteinhaltung gewertet wird. Andererseits war bei einem Teil der Vereinbarungen zum Befragungszeitpunkt deren Laufzeit noch nicht beendet, sodass sich noch nicht das definitive Ausmaß der Verstöße beziffern lässt.
In Westdeutschland haben die Arbeitgeber etwas häufiger (30 \%) ihre Zusagen nicht erfüllt als in Ostdeutschland (25\%). Ein Zusammenhang nach Betriebsgrößenklassen zeichnet sich in der bivariaten Verteilung ab. Im kleinsten Segment von 20-49 Beschäftigten geben die Betriebsräte in $28 \%$ der Fälle Verstöße an, bei den Betrieben mit über 2.000 Beschäftigen in $32 \%$. In den Segmenten dazwischen streuen die Anteile erratisch zwischen diesen Werten. Auch zwischen den Branchen streuen die Werte; die Kredit- und Versicherungswirtschaft stellt mit lediglich $14 \%$ gebrochenen Arbeitgeberzusagen einen Ausreißer nach unten dar.

\section{Multivariate Analyse}

Im Folgenden untersuchen wir, welche Faktoren die Wahrscheinlichkeit beeinflussen, dass Bündniszusagen der Arbeitgeber nicht eingehalten werden. Wir schätzen hierzu zwei logistische Regressionsmodelle. Beide Modelle haben die dichotom kodierte Information zur abhängigen Variable, ob beschäftigungsrelevante Zusagen des Betriebs eingehalten wurden. ${ }^{8}$ Das erste Modell umfasst alle Bündnisbetriebe, das

\footnotetext{
Beim Vergleich dieser Anteilswerte ist zu beachten, dass nicht für alle Betriebe alle Beschäftigungssicherungsmaßnahmen und Zusagen in Betrach kommen. Z.B. werden nur Ausbildungsbetriebe die Übernahme der Ausgebildeten vereinbaren.

8 Dem Fall des arbeitgeberseitigen Wortbruchs folgt jedoch nicht zwingend der Bruch des gesamten betrieblichen Regelungspakets.
} 
Tabelle 3: Einflussfaktoren auf die Stabilität Betrieblicher Bündnisse für Arbeit - logistische Regressionen ${ }^{1}$

Erklärende Variablen

Branche: Grundstoffe und Produktionsgüter

Investitions- und Gebrauchsgüter

Verbrauchsgüter

Baugewerbe

Verkehr und Nachrichten

Referenz: sonstige Dienstleistungen

1. Betrieb ist nur von Wirtschaftskrise betroffen

2. nur von sonstiger Krise betroffen

3. von Wirtschaftskrise und sonstiger Krise betroffen

Referenz Modell 1: nicht betroffen; Referenz Modell 2: nur von

sonstiger Krise betroffen

Form der Vereinbarung: nur mündliche Arbeitgeberzusage $\quad 0,169$

Referenz: schriftliche Absprache (Betriebsvereinbarung,

Regelungsabrede, tarifliche Regelung)

Beziehung Arbeitgeber - Betriebsrat: Behinderung Mitwirkung des BR durch AG: häufig

Behinderung Mitwirkung des BR durch AG: nie

Referenz: Behinderung Mitwirkung des BR durch AG: manchmal

Zusagen des Arbeitgebers:

1. Ausschluss betriebsbedingter Kündigungen

2. Erhalt der gegenwärtigen Belegschaftsstärke

4. Erhalt des Standorts / Garantie von Produktlinien

\section{Geplante und umgesetzte Maßnahmen zur}

Beschäftigungssicherung:

1. Abstriche beim Entgelt

6. Personelle und organisatorische Maßnahmen

schriftliche Vereinbarung anlässlich der Krise nicht abgeschlossen

oder verändert

Form der schriftlichen Vereinbarung: Betriebsvereinbarung
Logistische Regression 1:

Alle Betriebe mit

Arbeitgeberzusagen

b-Koeffizienten

$-0,000 * *$

$(0,000)$

$-0,583^{*}$

$(0,263)$

0,023

$(0,234)$

$-0,115$

$(0,255)$

$-0,211$

$(0,358)$

0,275

$(0,320)$

$-0,095$

$(0,208)$

0,263

$(0,306)$

$0,455+$

$(0,256)$

$(0,182)$

$0,525^{*}$

$(0,245)$

$-0,811 * *$

$(0,172)$

$-0,405^{*}$

$(0,174)$

$-0,472$ * *

$(0,170)$

0,039

$(0,196)$

0,623 * *

$(0,178)$

$0,273+$

$(0,164)$
Logistische Regression 2: Alle Krisenbetriebe mit schriftlichen Vereinbarung(en) zur Beschäftigungssicherung und Arbeitgeberzusagen

b-Koeffizienten

$-0,001$

$(0,000)$

$-0,343$

$(0,544)$

0,540

$(0,540)$

$-0,252$

$(0,657)$

$-0,946$

$(0,991)$

$-0,946$

$(0,831)$

$-0,853$

$(0,595)$

$-0,509$

$(0,646)$
Form: Tarifvertrag

Form: sonstige Vereinbarung / Regelungsabrede

$-0,722+$

$(0,396)$

0,073

$(0,473)$

$-0,071$

$(0,398)$

1,034

$(0,577)$

0,141

$(0,386)$

0,623

$(0,388)$

$-0,252$

$(0,372)$

$-0,028$

$(0,434)$

$0,988^{*}$

$(0,471)$

0,260

$(0,488)$

Notfallklausel vorhanden und genutzt?

$1,643^{*}$

$(0,638)$

1. vorhanden \& genutzt

0,252

. vorhanden aber nicht genutzt

$(0,405)$

Referenz: 3. nicht vorhanden

$-0,861+$

$(0,439)$

die Vereinbarung bezahlen. (Trifft zu)

0,377

$(0,482)$

erkauft worden. (Trifft zu)

$-1,025$

Konstante

$-0,683+$

$(0,355)$

Betriebe ( $\mathrm{n}$ )

1.016

$(1,077)$

chi $^{2}$

125,981 **

291

bic

1238,921

$77,754^{* *}$

442,908

Pseudo-R² (McFadden)

1 Die abhängige Variable nimmt den Wert 1 an, wenn Zusagen des Arbeitgebers 2009 oder 2010 teilweise oder gänzlich nicht eingehalten wurden, sonst den Wert 0. Die erklärenden Variablen sind bis auf Betriebsgröße dichotom kodiert. Arbeitgeberzusagen, geplante und umgesetzte Maßnahmen sowie Form der schriftlichen Vereinbarung sind Mehrfachnennungen.

Weitere nicht ausgewiesene erklärende Variablen sind in den Modello bunden (nur Modell 1): Lage des Betriebs in Ostdeutschland; Betrieb ist mittel oder stark exportabhäng dernis der Gewerkschaften: 1. nein 2. nein, da kein Tarifvertrag gegenüber Referenz: 3. ja, Zustimmungserfordernis vorhanden (nur Modell 2).

Weitere Arbeitgeberzusagen: Erhalt von Ausbildungskapazitäten/Übernahme, Neueinstellungen, Investitionen am Standort.

Weitere Maßnahmen zur Beschäftigungssicherung: Kurzarbeit, Abbau Arbeitszeitkonten/Aufbau Zeitschulden, Veränderung sonstige Arbeitszeit, Regelungen zur Urlaubsnahme, Einschnitte bei betrieblichen Sozialleistungen.

B-Koeffizienten sind logarithmierte Wahrscheinlichkeitsänderungen auf den Eintritt der abhängigen Variable unter Kontrolle der restlichen unabhängigen Variablen. In Klammer wird der Standardfehler ausgewiesen. Die Sterne markieren das Signifikanzniveau $\left(+p<0,10 ;{ }^{*} p<0,05\right.$; $\left.{ }^{* *} p<0,01\right)$. 
zweite nur Betriebe mit schriftlichen Vereinbarungen, sofern sie von einer Krise betroffen sind (Tabelle 3). Beide Modelle zeigen sich als mäßig aussagekräftig. Interpretiert man McFaddens Pseudo-R ${ }^{2}$ als Anteil der erklärten Varianz, so läge dieser im ersten Modell bei rund $11 \%$ und im zweiten bei $25 \%$. Die Interpretation dieser Gütemaße wird jedoch, besonders im zweiten Modell, durch die überschaubare Fallzahl bei einer hohen Zahl erklärender Variablen eingeschränkt.

\subsection{BETRIEBLICHE ARBEITS- BEZIEHUNGEN}

Vor allem die Beziehung zwischen Arbeitgeber und Betriebsrat übt einen signifikanten Effekt auf die (logarithmierte) Wahrscheinlichkeit aus, dass der Arbeitgeber Zusagen eines betrieblichen Bündnisses nicht einhält. Wird der Betriebsrat nach eigener Einschätzung häufig durch den Arbeitgeber behindert und kann somit, wie in Hypothese 1 ausgeführt, das Verhältnis als wenig vertrauensvoll charakterisiert werden, erhöht sich in beiden Modellen signifikant die Wahrscheinlichkeit, dass der Arbeitgeber seine Zusagen verletzt. Umgekehrt verringert sich diese Wahrscheinlichkeit in beiden Modellen signifikant (in einem Modell jedoch nur auf dem 10-\%-Niveau), wenn die Mitwirkung des Betriebsrates nie durch den Arbeitgeber behindert wird. Eine konflikthafte Beziehung zwischen Arbeitgeber und Betriebsrat zeigt in unserem Modell den stärksten Einfluss auf die Verlässlichkeit der gemachten Zusagen zur Beschäftigungssicherung.

Die Schätzwerte des zweiten Modells, das alle krisenbetroffenen Betriebe mit schriftlichen Vereinbarungen umfasst, bekräftigen diesen Zusammenhang. Es zeigt sich, dass Bündnisse, die vom Betriebsrat als ausgewogen eingeschätzt werden, da beide Betriebsparteien „einen fairen Preis für die Vereinbarung bezahlen“" mussten, die Wahrscheinlichkeit eines Verstoßes (Hypothese 2) senken. Die Symmetrie der getauschten Inhalte wirkt sich - wenngleich hier nur auf dem 10-\%-Niveau signifikant - positiv auf die Verlässlichkeit des BBA aus.

\subsection{INHALTE}

Im ersten Modell, das alle Bündnisbetriebe umfasst, sind ferner bei den Inhalten der
Vereinbarungen signifikante Einflüsse auf die Zusagebeständigkeit zu beobachten. So korrelieren die Zusagen der Arbeitgeber, betriebsbedingte Kündigungen auszuschließen und die gegenwärtige Belegschaftsstärke zu erhalten, negativ mit dem arbeitgeberseitigen Bruch der Zusage. Die in Hypothese 3 in Abschnitt 2 dargelegten Überlegungen lassen sich somit nicht bestätigen. Dieses Ergebnis lässt sich dahingehend interpretieren, dass der Betriebsrat bei diesen Regelungsinhalten über vergleichsweise weitreichende Mitbestimmungsmöglichkeiten verfügt und die Betriebe bei Nichteinhaltung der Zusagen Konflikte fürchten müssen. Bei Entlassungen ist die Zustimmung des Betriebsrats einzuholen. Zudem ist der Verzicht auf betriebsbedingte Kündigungen gegenüber den anderen Arbeitgeberzusagen auch individualrechtlich einklagbar.

Wenn die Beschäftigten Abstriche beim Entgelt hinnehmen, steigt die Wahrscheinlichkeit, dass Arbeitgeber ihre Zusagen nicht einhalten. Vermutlich dürften solche Vereinbarungen mit derartigen Einschnitten nur in betrieblichen Situationen mit besonders prekärer Wirtschaftslage oder konfliktären oder asymmetrischen Beziehungen zwischen den Betriebsparteien durchzusetzen sein. Diese drei Aspekte, die Krisenbetroffenheit, die Behinderung von Mitwirkungsrechten und die Asymmetrie des Tauschs, konnten wir direkt prüfen. So haben wir oben gezeigt, dass das Verhältnis zwischen den Betriebsparteien und die Einschätzung über die Gleichwertigkeit des Tauschs einen signifikanten Einfluss auf die Zusagebeständigkeit ausüben.

\subsection{AUSGANGSLAGE UND KONTEXT- BEDINGUNGEN}

Weder für die Krisenbetroffenheit noch für die Schriftform lassen die Schätzwerte entgegen unseren Annahmen einen signifikanten Effekt erkennen. Lediglich für den Fall, dass Betriebe sowohl von der Wirtschaftskrise als auch einer sonstigen betrieblichen Krise betroffen sind, deutet sich eine höhere Wahrscheinlichkeit an, dass Arbeitgeberzusagen nicht eingehalten werden (Hypothese 4). Der entsprechende Koeffizient ist jedoch nur auf dem 10-\%-Niveau signifikant, was die Sicherheit der Aussage einschränkt. Im zweiten Modell mit den Betrieben mit nur schriftlichen Vereinbarungen untersuchen wir zudem, ob diese anlässlich der Krise abgeschlossen oder verändert wurden (Hypothese 5). Auch hier lässt sich kein Zusammenhang zur Krisensituation ermitteln. ${ }^{9}$

In Modell 2 bestätigt sich der in Hypothese 6 beschriebene Zusammenhang, dass das Vorhandensein einer Notfallklausel die Wahrscheinlichkeit erhöht, dass diese genutzt wird und somit im Verlauf der Wirtschaftskrise häufiger arbeitgeberseitige Zusagen nicht erfüllt wurden.

Nicht bestätigen lassen sich unsere Erwartungen über den Einfluss der Form der Vereinbarung, ob schriftlich oder mündlich (Hypothese 8). Es zeigt sich im Vergleich der beiden Modelle, dass die Zusammenhänge in der Gruppe der nur schriftlichen Krisenbündnisbetriebe weitgehend mit denen im ersten Modell übereinstimmen. Lediglich im zweiten Modell, in der Gruppe der Betriebe mit nur schriftlichen Vereinbarungen, bestätigt sich der von uns in Hypothese 9 unterstellte Zusammenhang. Zusagen werden seltener eingehalten, wenn die Vereinbarung in Form eines Tarifvertrages geschlossen wurde.

Einen signifikanten Einfluss auf die Bündnisstabilität üben die Betriebsgröße und der Brancheneffekt aus. In größeren Betrieben sinkt die Wahrscheinlichkeit des Verstoßes. Das erste Modell liefert für das Grundstoff- und Produktionsgütergewerbe einen negativen Effekt auf die Wahrscheinlichkeit, dass Arbeitgeberzusagen geringeren Bestand haben.

Betrachtet man nun die restlichen erklärenden Variablen, so trägt deren Mehrheit nicht zur Erklärung des arbeitgeberseitigen Zusagenbruchs bei. Weder die Exportabhängigkeit noch die geografische Lage, die Anbindung an den Flächentarifvertrag oder das Zustimmungserfordernis der zuständigen Gewerkschaft (Hypothese 7) zeigen einen Effekt.

Zusammenfassend lässt sich sagen, dass vor allem die Beziehungen zwischen Arbeitgeber und Betriebsrat in beiden Modellen einen entscheidenden Erklärungsanteil für die Stabilität von BBA haben.

9 Die Krisenbetroffenheit der Betriebe wurde von uns in hier nicht vorgestellten Regressionsmodellen ebenfalls mit anderen Indikatoren wie der Auftrags- und Umsatzlage überprüft. Auch hier zeigte sich das gleiche Bild. 
Zusagen und der Wirtschaftskrise feststellen. Die Wahrscheinlichkeit, dass Zusagen gebrochen werden, unterscheidet sich nicht zwischen den bereits vor und den während der Krise abgeschlossenen Vereinbarungen. Insofern zeigt sich bei den Beschäftigungsbündnissen eine überraschende Stabilität gegenüber äußeren Einflüssen.

Eine Rolle für die Stabilität der Bündnisse haben die betrieblichen Arbeitsbeziehungen. Ein gutes Verhältnis zwischen den Betriebsparteien erhöht die Beständigkeit der Arbeitgeberzusagen. Im Umkehrschluss dürfte es für das Vertrauen des Betriebsrats in das Management nicht unbedeutsam sein, inwieweit die getroffenen Vereinbarungen vom Arbeitgeber auch eingehalten werden, wie Nienhüser und Hoßfeld (2010a, 2010b) zeigen. Dieser Zusammenhang steht im Einklang mit dem Befund, dass unter den Bedingungen beidseitig professionalisierter Arbeitsbeziehungen in Großbetrieben ein hohes Maß an sozialpartnerschaftlicher Kooperation und Co-Management besteht (Rehder 2003).
Machte Rehder (2006) die im CoManagement drohende Gefahr eines Legitimationsdefizits im Verhältnis des Betriebsrats gegenüber den Beschäftigten aus, so zeigt unsere Analyse, dass sich betriebliche Bündnisse auch unter erschwerten Bedingungen bewährten. Sie konnten ihre Schutzwirkung für die Beschäftigten vor allem dann entfalten, wenn das Verhältnis zwischen den Betriebsparteien als konfliktarm anzusehen war. Dies muss jedoch vor dem Hintergrund gesehen werden, dass die generelle Stabilität der Bündnisse als durchaus steigerungsfähig eingeschätzt werden kann. Immerhin hielt knapp ein Drittel der Betriebe die Zusagen zumindest partiell nicht ein.

10 Eine Übertragung des Anteils der Betriebe mit nicht eingehaltenen Arbeitgeberzusagen auf den Anteil der vorzeitig beendeten Bündnisse sollte vermieden werden. Bündnisse können trotz nicht eingehaltener Arbeitgeberzusagen fortbestehen. Zudem wurden die Bündnisse innerhalb ihrer Laufzeit erfasst, daher besteht die Möglichkeit weiterer Brüche in der Zukunft.

doch keinen Zusammenhang zwischen der arbeitgeberseitigen Einhaltung gemachter

\section{LITERATUR}

Bellmann, L./Gerlach, K./Meyer, W. (2008): Company-Level Pacts for Employment, in: Jahrbücher für Nationalökonomie und Statistik 228 $(5+6)$, S. 533-553

Berthold, N./Brischke, M./Stettes, O. (2003): Betriebliche Bündnisse für Arbeit - Gratwanderung zwischen Tarifbruch und Tariftreue, in: ORDO Jahrbuch für die Ordnung von Wirtschaft und Gesellschaft 54, S. $175-193$

Bogedan, C./Brehmer, W./Herzog-Stein, A. (2009): Betriebliche Beschäftigungssicherung in der Krise. Eine Kurzauswertung der WSIBetriebsrätebefragung 2009, WSI-Report 1, Düsseldorf

Büttner, R./Kirsch, J. (2005): Bündnisse für Arbeit im Betrieb, Düsseldorf Haipeter, T. (2009): Tarifregulierung zwischen Fläche und Betrieb: Koordinierung und Praxis in der Chemie- und der Metallindustrie, in: WSI-Mitteilungen 62 (4), S. 185-192

Hassel, A./Rehder, B. (2001): Institutional Change in the German Wage Bargaining System - The role of Big Companies, MPIfG Working Paper 01/09, Köln

Herzog-Stein, A./Seifert, H. (2010): Stabile Beschäftigung durch flexible Arbeitszeiten, WSI-Diskussionspapier 169, Düsseldorf

Hübler, O. (2005): Sind betriebliche Bündnisse für Arbeit erfolgreich?, in: Jahrbücher für Nationalökonomie und Statistik 225 (6), S. 630-652 Kaden, T. (2003): Individuelle Bündnisse für Arbeit und Günstigkeitsprinzip, Frankfurt/Main

Kocher, E. (2009): Effektive Mobilisierung von Beschäftigtenrechten. Das Arbeitsrecht in der betrieblichen Praxis, Forschungsmonitoring, Düsseldorf Massa-Wirth, H. (2007): Zugeständnisse für Arbeitsplätze? Konzessionäre Beschäftigungsvereinbarungen im Vergleich Deutschland - USA, Berlin
Nienhüser, W./Hoßfeld, H. (2010a): Vertrauen und Verbetrieblichungsneigung von Managern und Betriebsräten. Ergebnisse einer empirischen Erhebung - Teil 1, in: Sozialer Fortschritt 59 (8), S. 219-225

Nienhüser, W./Hoßfeld, H. (2010b): Vertrauen und Verbetrieblichungsneigung von Managern und Betriebsräten. Ergebnisse einer empirischen Erhebung - Teil 2, in: Sozialer Fortschritt 59 (9), S. 227-234 Rehder, B. (2003): Betriebliche Bündnisse für Arbeit in Deutschland. Mitbestimmung und Flächentarif im Wandel, Frankfurt am Main Rehder, B. (2006): Legitimitätsdefizite des Co-Managements. Betriebliche Bündnisse für Arbeit als Konfliktfeld zwischen Arbeitnehmern und betrieblicher Interessenvertretung, in: Zeitschrift für Soziologie 35 (3),

\section{S. $227-242$}

Rottleuthner, H. (1987): Einführung in die Rechtssoziologie, Darmstadt Sachverständigenrat zur Begutachtung der gesamtwirtschaftlichen Entwicklung (SVR) (2007): Das Erreichte nicht verspielen, Jahresgutachten 2007/2008, Wiesbaden

Schäfer, C. (2008): Die WSI-Betriebsrätebefragung 2007 - Methode und ausgewählte Ergebnisse, in: WSI-Mitteilungen 61 (6), S. 291-296 Seifert, H. (2002): Betriebliche Bündnisse für Arbeit - Beschäftigen statt entlassen, in: Seifert, H. (Hrsg.): Betriebliche Bündnisse für Arbeit. Rahmenbedingungen - Praxiserfahrungen - Zukunftsperspektiven, Berlin, S. 65-85

Seifert, H./Massa-Wirth, H. (2004): Betriebliche Bündnisse für Arbeit nur mit begrenzter Reichweite?, in: WSI-Mitteilungen 57 (5), S. 246-254 Zapf, I./Brehmer, W. (2010): Flexibilität in der Wirtschaftskrise: Arbeitszeitkonten haben sich bewährt, IAB-Kurzbericht 22, Nürnberg 